\title{
Herzinsuffizienz
}

\section{Manchmal gibt es Besseres als ACE-Hemmer}

_ Die Leitlinien der Europäischen Society of Cardiology (ESC) zur Herzinsuffizienz (HF) haben 2016 entscheidende Veränderungen erfahren. Das betrifft sowohl die Einteilung der HF als auch die Therapieempfehlungen für Patienten mit schwer behandelbaren Formen einer Herzinsuffizienz mit reduzierter Ejektionsfraktion (HFrEF).

\section{In schweren Fällen: \\ ARNI statt ACE-Hemmer}

Die Einteilung der Herzinsuffizienz wurde in den ESC-Leitlinien erweitert: Zwischen die klassischen Kategorien von HFrEF (linksventrikuläre Auswurffraktion $[\mathrm{LVEF}]<40 \%$ ) und $\mathrm{HFpEF}$
(LVEF $\geq 50 \%$ bei erhöhten natriuretischen Peptiden sowie strukturellen Herzveränderungen und/oder diastolischer Dysfunktion) sei die neue Klasse der HFmrEF eingefügt worden, berichtete Prof. Burkert Pieske, Berlin. HF-Patienten der neuen Kategorie haben eine „mäßig reduzierte“ („mid-range“) LVEF zwischen 40 und 49\%) erhöhte natriuretische Peptide sowie strukturelle Herzveränderungen und/oder eine diastolische Dysfunktion.

Geradezu als Paradigmenwechsel bezeichnete Pieske die Empfehlung, HFrEFPatienten, die unter der Kombination von Diuretikum, ACE-Hemmer, Betablocker und Mineralokortikoid/Aldosteron-Re-
zeptor-Antagonist symptomatisch blieben, zukünftig nicht mehr mit einem ACE-Hemmer oder einem AngiotensinRezeptor-Blocker weiter zu behandeln. Stattdessen sollten diese Patienten auf die neue Substanzklasse der Angiotensin-Rezeptor-Neprilysin-Inhibitoren (ARNI) umgestellt werden. Einziger derzeit verfügbarer Vertreter dieser Wirkstoffklasse ist die Kombination von Sacubitril und Valsartan (Entresto ${ }^{\circ}$. In den europäischen und US-amerikanischen Leitlinien wurde eine Empfehlung der Klasse Ib für ARNI ausgesprochen.

Jan Groh

- Novartis Kardiologentage 2017,ESC-Guideline 2016: Und nun?"; Berlin, März 2017 (Veranstalter: Novartis)

\section{Diabetes mellitus Typ 2}

\section{Die Therapie zügig intensivieren}

— Nach Dr. Andreas Lueg, Hameln, muss die medikamentöse Diabetestherapie - nach Metformin - individuell erfolgen, so Lueg. Zwei Kriterien sind dem Diabetologen dabei wichtig: Hypoglykämien müssten vermieden werden, ebenso eine Gewichtszunahme.

DPP-4-Hemmer seien gewichtsneutral, SGLT-2-Hemmer und GLP-1-Analoga gewichtsreduzierend, wobei letztere subkutan appliziert werden und nicht peroral. Bei SGLT-2-Hemmern müsse die berufliche Situation des Patienten bedacht werden. Denn pro Tag müsse dieser zweimal häufiger auf die Toilette. Das spreche z. B. bei einem Busfahrer eher für einen DPP-4-Hemmer zusätzlich zu Metformin.

Im Vergleich zu Sulfonylharnstoffen (SH) haben DPP-4-Hemmer eine deut- lich längere Wirksamkeit, so Lueg. Das habe etwa eine Studie mit 624 Typ2 -Diabetikern ergeben. Eine Gruppe erhielt einen $\mathrm{SH}$ plus Metformin, die andere Metformin plus den DPP-4-Hemmer Sitagliptin (z. B. Xelevia oder Velmetia ${ }^{\oplus}$, eine Fixkombination mit Metformin).

In den ersten 18 Monaten sank der $\mathrm{HbA}_{1 \mathrm{c}}$-Wert in beiden Gruppen. Anschließend stieg er in der SH-Gruppe wieder an, während er unter dem DPP4-Hemmer weiter zurückging und dann über insgesamt 60 Monate stabil blieb [Pharmacol Res. 2015;100:127-34].

Die Therapie-Eskalation bei Typ2-Diabetikern werde häufig unnötig verzögert, warnte Lueg. Eine Verzögerung von ein bis zwei Jahren bedeute aber 30-50\% mehr kardiovaskuläre Er-

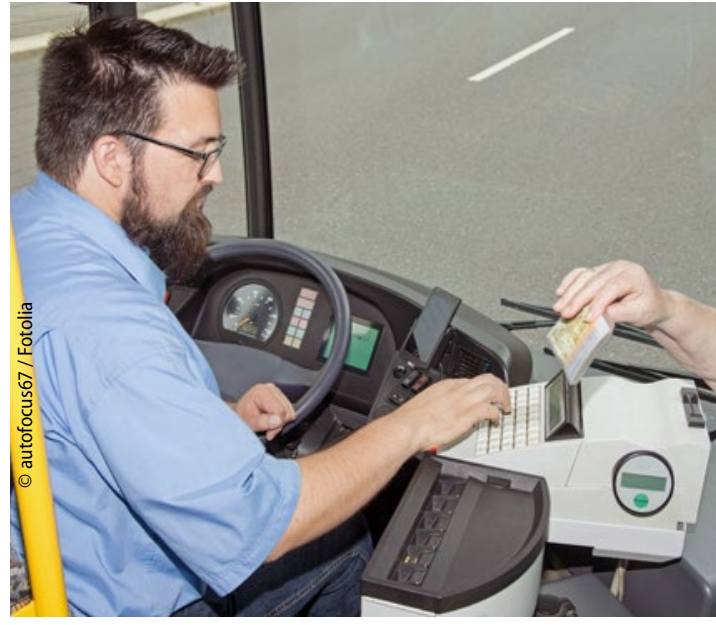

Die Diabetestherapie muss immer auch die berufliche Situation berücksichtigen.

eignisse [Cardiovascular Diabetology 2015;14:100].

Dr. Michael Hubert

- Symposium "Stoffwechselkonsequenzen der Adipositas mehr als Typ-2-Diabetes", DGIM 2017; Mannheim, April 2017 (Veranstalter: Berlin-Chemie) 